\title{
Numerical simulation of roll compaction of aerated powders
}

\author{
V. Esnault ${ }^{\mathrm{a}, \mathrm{b}}$, D. Heitzmann ${ }^{\mathrm{b}}$, M. Michrafy ${ }^{\mathrm{c}}$, D. Oulahna ${ }^{\mathrm{d}}$, A. Michrafy ${ }^{\mathrm{d}, *}$ \\ ${ }^{a}$ Laboratoire Navier, Ecole des Ponts ParisTech, 6/8 Avenue Blaise Pascal, Champs sur Marne 77455, France \\ ${ }^{\mathrm{b}}$ Lafarge Centre de Recherche, 95 rue de Montmurier-BP 15, F-38291 St Quentin Fallavier, France \\ c BEM, Bordeaux Management School, 680 cours de la Libération, F-33405 Talence, France \\ ${ }^{\mathrm{d}}$ Université de Toulouse, Mines Albi, CNRS, Centre RAPSODEE, Campus Jarlard, F-81013 Albi cedex 09, France
}

H I G H L I G H T S

- We explore the role of the air trapped into fine powders during roll compaction process.

- We determine density and velocity of the solid during the process, using Johansson model and mass conservation.

- We determine gas pressure distributions, using Darcy's law.

- We investigate effect of powder density and particle fragmentation on air pressure using Kozeny-Carman law.

- We discuss the initiation of instabilities based on the fluidization criterion and the impact of process parameters.

\section{A B S T R A C T}

Permeating air is known to have a negative impact on the roller compaction process, because the feed is destabilized by the flow of escaping gas, causing pressure to build-up and potentially damage compacts at release. Airflow during powder roller compaction and its effect on the rolling process are investigated in the rolling direction (1D), using an extension of the Johanson model for the solid. Fluid transport obeys Darcy's law, with permeability being a function of both material density and particle size, through the Kozeny-Carman relationship. In this modeling, the effect of the air pressure on the solid is neglected in the compaction zone. Assuming air at atmospheric pressure at the feeding angle and ignoring airflow through the gap, predictions of air pressure as a function of the rolling angle for bentonite material powder are presented and discussed. Results suggest the existence of two different stability zones within the operating conditions, where industrial systems could function without being affected by airflow effects. The model highlights the importance of the permeability/rotation speed ratio, which governs the proportion of air trapped in the compacts to the portion evacuated through the feed. We also investigate the effect of particle fragmentation during the rolling process. Finally, we provide guidelines for the scale-up of roller presses subjected to air flow issues, through a study of the effect of the system dimensions and rotation speed on the elimination of air.

In spite of the lack of available experimental data, this model allows for a better understanding of how air escapes by diffusing through the material during the rolling process, and opens interesting perspectives for the mitigation of its effect on the process.

Keywords:

Air pressure

Fragmentation

Porous media

Roll compaction

Stability

Scale-up

\section{Introduction}

Roll presses are routinely employed in various industrial processes, for example in the pharmaceutical and mineral industries. The powder is fed to the compaction zone either by gravitational feeding or by a screw feeder. The powder is then drawn between two counter-rotating rolls in the consolidation zone, where a high pressure is applied. In the pharmaceutical

\footnotetext{
* Corresponding author. Tel.: +335634931 62; fax: +33563493025

E-mail address: michrafy@mines-albi.fr (A. Michrafy).
}

industry, the process is used for dry granulation agglomeration of fine powder mixtures to improve flowability for direct compression. In the mineral industries, presses are used for grinding, as the particles are fractured into finer fragments by the high pressure generated.

The roller compaction process has been the object of several attempts at numerical modeling. The present work belongs to the 1-D model family. Conditions in the material are considered variable in the direction of flow only: a reasonable hypothesis when considering the geometry of the system. Notable 1-D models found in the literature include Johanson's model (Johanson, 1965), the slabs model (Dec et al., 2003) or the thin layers model 
(Peter et al., 2010). A good literature review of these models can be found in (Cunningham, 2005).

Recently, finite-element numerical simulations have allowed compaction to be modeled in 2-D (Dec et al. 2003, Michrafy et al. 2011b, Muliadi et al., 2012), or in 3-D (Cunningham et al., 2010, Michrafy et al., 2011a). However, 1-D models still present an interest for their relative simplicity and low computational cost. Their predictions are also found to be in good agreement with finite element simulations. 1-D model development thus seems a reasonable first step to model any characteristics of the rolling process.

We have chosen to adopt a variation of the Johanson model (Johanson, 1965), which stands as a reference in the domain. Recent applications can be found for comparison to experimental data (Bindhumadhavan et al., 2005), comparison to 2-D finite element simulations (Michrafy et al. 2011b, Muliadi et al., 2012), or modeling of the dynamic behavior of the press (Hsu et al. 2010).

The present work aims to extend existing models by including modeling of the air and solid interactions. Air has a negative effect on the press operation through two mechanisms:

- Compaction creates an air transport away from the compacted zone, through the feed zone (Vinogradov and Fedorchenko, 1961). This in turn generates a pressure gradient that can disturb the incoming flow. In the case of gravitational feeding, where particles are only subjected to their own weight, it can even result in the fluidization of the particles, which totally disorganizes the press feeding. The press starts to vibrate as cycles of fluidization/consolidation of the feed arise (Schwechten and Milburn, 1990). Screw feeding allows for greater tolerance to air flow but is eventually also disrupted by the pressure gradient (Johanson and Cox, 1989). Such instabilities are detected by significant fluctuations in roll pressure and torque, and in some cases by vibrations and a popping noise generating rattling in of the whole drive system (Dec, 1995).

- On the other hand, air that is not evacuated accumulates in the compact and also leads to a pressure build up that can destroy the compacts when they are ejected from the press (Dec, 1995).

Knowledge of the effect of air in the roll compaction is widespread and has been strongly emphasized in the literature. But studies have hardly ever gone beyond this assessment and tried to predict the air elimination and pressure build-up during the process more precisely. The work of Johanson and Cox (1989) is a notable exception. In this very short paper, they present an extension of Johanson's numerical model for solid compaction (Johanson, 1965) to predict air transport, and present some meaningful results, including some considerations on process stability.

More generally, the problem of the air trapped inside the fine powders and its escape by transport through the material is also encountered in other powder-handling processes, such as silo discharge and screw feeding. In contrast to the roller compaction process, the effect of air pressure in discharging or loading a silo has been relatively well studied. Different analyses of the dissipation of air from an aerated powder in a silo have been proposed (Coffey and Gremaud, 2003, Johanson, 1971, Johanson and Jenike, 1971, Murfitt and Bransby, 1980). These approaches were used for hopper design (Johanson and Jenike, 1971), or for exploring the effect of the geometry of the container and the length of time a given material takes to consolidate (Coffey and Gremaud, 2003).

In the rolling process, which is the object of this study, the situation is made even more complex by the considerable transformations the powder is subjected to: a high degree of compaction, particle fragmentation, etc. The disturbances are also closely linked to the dynamic of the system and most notably to the rolling speed.
The present study explores the role of the air transport in the feed powder during the rolling process through the analysis of created gas pressure distributions, with a simple, 1D, numerical model. We base our study on an extension of the well-known Johanson model for the solid phase (Johanson, 1965) for the prediction of the solid behavior, but a very similar approach could be adopted for any 1D model of roller compaction.

The transport equations of air in the powder are described, in the rolling direction, using Darcy's law. We also investigate the combined effect of powder density and particle fragmentation on air transport using the Kozeny-Carman law for permeability. Particle size evolution is fitted from experimental data.

We discuss the initiation of instabilities based on the fluidization criterion originally proposed by Johanson and Cox (1989). We also evaluate the impact of different process parameters, such as the combined effect of rolling speed and powder permeability. We give particular attention to the effect of the fragmentation of particles and of the dimension of the press, two parameters that, to our knowledge, have never been investigated.

\section{Solid and air flow numerical model}

\subsection{Solid behavior}

For the prediction of the evolution of solid properties, we have based our analysis on the classic Johanson model. An extensive description of this model can be found in Dec (1995) and Sommer and Hauser (2003) and here we shall only briefly recall the main characteristics of this model.

The geometry of the system is presented in Fig. 1a. Material is fed to the rolls at angle $\theta_{0}$. The position of the material can be characterized either by the rotation angle $\theta$ or the position
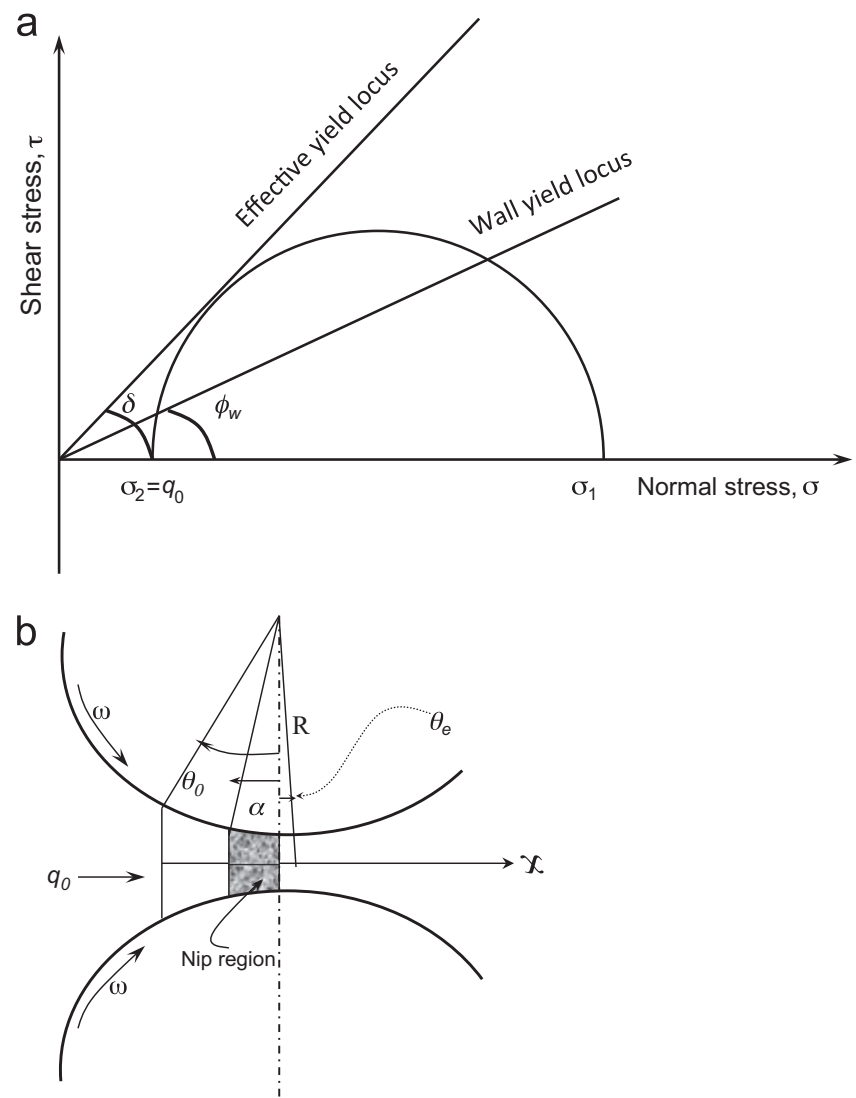

Fig. 1. (a) Effective and wall yield locus. (b) Roll press and nip angle. 
$x=R \sin \theta$. The gap is the zone where the distance of the rolls is minimal, at $\theta=0$. Materials are released shortly after the gap at the angle of release $\theta_{e}$.

The Johanson model we are basing our study upon only provides a model for the zone between $\theta=0$ and $\theta_{0}$. Modeling the release zone (between $\theta=0$ and $\theta_{e}$ ) is accessible to a 1D model (Cunningham, 2005), but good knowledge of the elastic properties of compacts is required, and phenomena that are hard to predict, such as slip or compact fracture, can occur.

\subsubsection{Bulk properties of the solid}

The powder behavior is assumed to be isotropic, frictional, cohesive and compressible and to obey the Jenike and Shield effective yield function (Jenike and Shield, 1959). The powder properties, internal friction angle $\delta$ and wall friction angle $\phi_{\mathrm{w}}$ shown in Fig. 1a, are measured using a direct shear test, originally proposed in (Jenike et al., 1960).

In the concept of the Johanson model (Johanson, 1965), the mean normal stress is defined as:

$\sigma_{\theta}=\left(\sigma_{1}(\theta)+\sigma_{2}(\theta)\right) / 2$

where $\sigma_{1}$ and $\sigma_{2}$ are major and minor principal stresses.

The mean normal stress $\sigma_{0}$ at the feed angle $\theta_{0}$ of application of the known stress applied in the flow direction, $q_{0}$, and is given by

$\sigma_{0}=q_{0}(1-\sin (\delta))$

The feed angle $\theta_{0}$ is assumed to be:

$\theta_{0}=\left(\phi_{w}+\arcsin \left(\sin \left(\phi_{w}\right) / \sin (\delta)\right)\right) / 2$

and its computation is possible when the angle of wall friction $\phi_{\mathrm{w}}$ and the internal friction angle $\delta$ are known. This, however, depends exclusively on the material data and the wall friction parameters. This is one of the differences with finite element modeling (Cunningham et al., 2010, Michrafy et al., 2011a, Michrafy et al., 2011b) where the feeding angle could be chosen independently of powder properties. For simplification purposes, we assume in the rest of this study that the feeding angle corresponds to this angle $\theta_{0}$.

\subsubsection{Pressure-density relationship of the solid}

The feed powder is drawn between two counter-rotating rolls in the compaction zone, where a high pressure is applied to form a strip that has the dimensions of the roll gap. Drawing of the powder into the press and densification mechanisms are based on the concept of the nip angle $\alpha$, which defines two contiguous zones between rolls, represented in Fig. 1b. Before the nip angle, in the area named the slip region, the powder is transported by relative sliding on the roll and obeys the Jenike's yield criteria, and a slip condition at the surface of the rolls. Once the nip angle is reached, the powder is assumed to be drawn by sticking to the roll surface and to be compacted by deformation under the high roll pressure near the gap. In this region, stress evolution can be calculated, assuming adherence of the material on the walls and a behavior law linking density to the mean stress. In this study we adopt the following form:

if $\sigma_{\theta} \leq \sigma_{c}$ then $\rho_{\theta}=\rho_{0}$

if $\sigma_{\theta}>\sigma_{c}$ then $\rho_{\theta}=\rho_{0}\left(\frac{\sigma_{\theta}}{\sigma_{c}}\right)^{1 / \beta}$

$\rho_{0}$ is the density at the feeding angle (we further assume it to be equal to the tapped density), $\sigma_{c}$ a characteristic stress value. The constant $\beta$ is a material property representing the compressibility of the powder and is determined by a simple compression test using a tablet press.

Johanson adopted a law similar to (4b), but for all stress values. Condition (4a) was adopted here to prevent the density from dropping to nonphysical small values for small stresses. But in Johanson's work, relation (4b) plays a role only in the stick region, so the two approaches are strictly identical for the solid solution as long as $\sigma_{\theta} \geq \sigma_{c}$ in the stick region.

The solid material is considered incompressible. The reduction of the volume under pressure is only due to the reduction of the porosity $\phi$ defined as:

$\phi=1-\left(\frac{\rho_{\theta}}{\rho_{s}}\right)$

where $\rho_{s}$ is the true density of the solid. In Eq. (5), the porosity is, of course, a function of the rotating angle $\theta$.

\subsubsection{Pressure distribution of the solid}

In the Johanson model, the pressure between rolls is computed as follows (Johanson, 1965): first, two solutions are calculated for the stress gradient in the material, either assuming that slip occurs or does not occur on the roll surface. The "slip" solution describes the situation in the "slip zone", while the "stick" solution describes the situation in the nip zone. The nip angle is determined using a continuity argument on the stress gradient, and the stress solution for both zones can then be deduced. Finally, the pressure distribution between rolls is computed by combining the two solutions before and after the nip angle.

One important property of this solution is that it does not depend on the roll speed.

\subsubsection{Solid velocity in rolling direction}

The determination of the solid speed $v_{s}$ comes through the conservation of material mass. As the solution is in steady state, the quantity of solid is conserved in any Eulerian slab of material. In an equivalent manner, the flux of solid is constant through any given fixed surface in a cross-section of the rolling direction:

$(S+2 R(1-\cos (\theta)))(1-\phi(\theta)) v_{s}=A$

where $S$ is the gap, $R$ the roll radius, $v_{S}$ the solid velocity and $A$ a constant.

The constant $A$ can be determined in the nip zone from the roll rotation speed $\omega$ in rpm, as there is no slip between the roll and the material. For instance, at the gap $(\theta=0)$, we have:

$v_{S}(0)=\omega R$

which, once injected into Eq. (6), yields:

$v_{S}(\theta)=\omega S R(1-\phi(0)) /((S+2 R(1-\cos (\theta)))(1-\phi(\theta)))$

This expression can similarly be written in a shorter form as:

$v_{s}(\theta)=\omega f(\theta)$

where $f(\theta)=S R(1-\phi(0)) /((S+2 R(1-\cos (\theta)))(1-\phi(\theta)))$

\subsection{Gas pressure in the porous medium}

We now want to establish the gas pressure field in the powder. Establishing a solution for gas pressure from the solid solution supposes that the effect of the gas on the solid can be neglected. In poromechanics, it is well established that the effect of the fluid on the porous solid can be neglected when fluid pressure (and its variations) are small compared to solid stress (Coussy, 2004).

This assumption is true by several orders of magnitude between the rolls, where solid stresses reach values up to $100 \mathrm{MPa}$ or more, while gas pressures are of the order of magnitude of atmospheric pressure $(100 \mathrm{kPa})$. Thus the effect of the gas on the solid can be neglected.

Before the rolls, or after the release angle, this assumption does not hold, and the gas can have a possibly harmful effect on the 
solid (instability generation in the feed, damaging the compact at release).

We believe the procedure described here is similar to the one used by Johanson and Cox (1989). However, the description of the model for gas transport in that short paper is very succinct, preventing us from certifying that our approach is strictly similar to his.

\subsubsection{Continuity equation of the gas model}

First, a relation on the gas velocity $v_{g}$ is established from the gas conservation in any Eulerian slab, in a similar way to Eq. (6) for the solid:

$(S+2 R(1-\cos (\theta))) \phi(\theta) \rho_{g} v_{g}=$ const.

where $\rho_{g}$ is the density of the gas. In Eq. (9), $v_{g}$ represents the superficial velocity of gas.

\subsubsection{Constitutive equations of gas}

First, the gas is assumed to be ideal and isothermal (i.e. density $\rho_{g}$ is proportional to the gas pressure $P$ ). The variation in the temperature of the gas during the process is neglected here. Expected variations of temperature that do not exceed a few dozen Kelvin, are in any case insufficient to have any significant effect on the gas density.

With this assumption, Eq. (9) becomes:

$P(\theta)(S+2 R(1-\cos (\theta))) \phi(\theta) \quad v_{g}=$ const.

Secondly, the pressure gradient and gas velocity are related through Darcy's law, which can be written as:

$\phi(\theta)\left(v_{g}-v_{S}\right)=\frac{K(\phi)}{\mu_{g}} \frac{\partial P}{\partial x}$

where $K$ is the permeability of the powder depending on the local porosity and hence the position $\theta$. The constant $\mu_{g}$ is the gas viscosity.

Eliminating the gas velocity between (10) and (11), we reach:

$\left.P(\theta)(S+2 R(1-\cos (\theta))) \quad \phi(\theta) v_{S}(\theta)+\frac{K(\phi)}{\mu_{g}} \frac{\partial P}{\partial x}\right)=B$

where $B$ is a constant.

Knowledge of the permeability $K(\phi)$ is required to compute the gas pressure $P$. Its values can be determined experimentally for any powder for various porosities, and fitted by an empirical law. This was the approach chosen by Johanson and Jenike (1971). For this study, we prefer to adopt the widely used Kozeny-Carman relationship:

$K(\phi)=\frac{\phi^{3} D^{2} p}{180(1-\phi)^{2}}$

with

$D_{p}=\frac{6 V_{p}}{S_{p}}$

where $D_{p}$ is an average characteristic size of particles, calculated from the ratio between the average characteristic volume $V_{p}$ and the average characteristic surface $S_{p}$.

Darcy's law (11) is very widely used to model fluid diffusion through a porous solid. It applies as long as the gas flow is slow enough to be considered laminar. For higher speeds, a turbulent drag term in the square of the gas speed has to be considered (Forchheimer's law).

Dullien (1992), based on an extensive literature review, suggests that the field of application of Darcy's and Forchheimer's laws can be evaluated by calculating an appropriate Reynold's number $R_{e}=\frac{\rho_{g} v_{g} D_{p}}{\mu_{g}}$. Darcy's law could safely be considered to be applicable as long as $R_{e}$ is lower than 2 or 4 .

For fine powders and small systems, this relation is always verified. An evaluation of $R_{e}$ for $D_{p}=20 \mu \mathrm{m}$ and air at $20^{\circ} \mathrm{C}$ indicates that Darcy law's is applicable if $v_{g}$ is lower than 2 or $4 \mathrm{~m} \mathrm{~s}^{-1}$. For a $100 \mathrm{~mm}$ diameter press, the superficial speeds of the rolls, which are of the same order of magnitude as the gas speeds, will not reach such a value unless the rolls are turning at $400-800 \mathrm{rpm}$, which is unrealistic. This limitation should be kept in mind, though, when considering either bigger systems (increased $v_{g}$ or coarser powders).

The use of Eq. (13) is widely documented (Dullien, 1992), and is certainly a good choice for permeability prediction in the absence of further characterization of the powder. It also has the great advantage of introducing the dependence of the permeability in the particle size in a comprehensive way. From there, particle size can either be considered constant throughout the process, or expressed as a function of either the porosity or the stress in the grains, in the case of the reduction of particle sizes for brittle material during the rolling process.

The equations for the gas flow during the roll compaction process are completed by two boundary conditions:

$P\left(\theta_{0}\right)=P_{a t m}$

$\left.\frac{\partial P}{\partial x}\right|_{\theta=0}=0$

The first boundary condition consists in assuming that the gas is at atmospheric pressure at the feeding angle. In the case of a closed feeding system this might not be the case, however. If the air has to permeate through the material between the entry angle and any point at the atmospheric pressure, this could generate a pressure gradient that raises the pressure at entry beyond this value. One should then couple the present model with a model of the permeation through the feeding system.

With the second boundary condition, we assume that the pressure gradient is null at the gap. This corresponds to the situation where the flow of the gas is forced to be all opposite to the solid flow.

This assumption is acceptable as long as the quantity of gas escaping through the compacted material between $\theta=0$ and the release angle $\theta_{e}$ can be neglected. In the case of full dense material at the exit $(\phi(\theta=0)=0)$,the boundary condition (15) is verified. But in our case, the porosity at the exit is as 0.2 , which intuitively, can suggest the escape of a small quantity of gas toward the exit. However, for this study, Eq. (15) is a good approximation as long as condition (16) is verified. This relationship is a comparison between the gas flux due to Darcean transport through the compact to the release angle and the flux of gas entrained with the solid.

$\gamma=\frac{-\frac{K}{\mu_{g}} \frac{P-P_{a t m}}{R \sin \theta_{e}}}{\phi \omega R} \ll 1$

Assuming $\theta_{e}=-3^{\circ}$, a value comforted by the literature (Cunningham, 2005), relation (16) is well verified in most of the simulations presented in this study. Exceptionally, for small $R$ and high $\frac{K}{\omega}$ ratios, gamma $(\gamma)$ values as high as 0.3 are obtained. Condition (15) might be a bad approximation in those cases, and a modeling of the behavior in the release zone might be required to better account of the air flow.

\subsubsection{Combined effects of permeability and roll speed} on the gas pressure

One aspect concerns the relative role of permeability and roll rotation speed on the gas pressure described in the above model (Eq. (12)). Indeed, one can see in the equations that the rotation speed of the rolls and the permeability of the powder play a very similar role.

If we consider Eq. (8b), we can clearly see that $v_{s}$ is proportional to the rotation speed of the rolls. Using this expression in Eq. (12), 
Table 1

Powders properties.

\begin{tabular}{llllll}
\hline Powder & Internal friction $\delta\left(^{\circ}\right)$ & Wall friction $\phi_{w}\left({ }^{\circ}\right)$ & Compressibility $\beta$ & Blaine Specific Surface BSS $\left(\mathrm{cm}^{2} / \mathrm{g}\right)$ & Particle size $(\mu \mathrm{m})$ \\
\hline Bentonite & 39 & 32 & 7.84 & 8000 & 2.84 \\
Limestone & 40 & 35.75 & 13.05 & 170 & 2640 \\
\hline
\end{tabular}

and dividing all by $\omega$, a simple derivation according to $x$ yields:

$\frac{\partial}{\partial x}\left[P(\theta)(S+2 R(1-\cos (\theta)))\left(\phi f(\theta)+\frac{K(\phi)}{\mu_{g} \omega} \frac{\partial P}{\partial x}\right)\right]=0$

As previously stated, the permeability is calculated from the density (or porosity $\phi$ ) computed from the Johanson solution of stress for the solid, which does not depend on the roll rotation speed. It can now readily be seen that the solution $P$ of Eq. (17) is going to depend on $K(\phi)$ and rotation speed only through the ratio $K(\phi) / \omega$. In other words, the model predicts that a proportional variation of the permeability and the rotation speed of the roll are going to lead to the same calculated gas pressure, anywhere in the system.

\subsubsection{Definition of stability thresholds}

The dimensionless pressure gradient at the entry is defined as $\mathrm{DPG}=\frac{\partial P}{\partial x} / \rho_{S} g$ where $\rho_{S}$ is the true particles density and $g$ the gravity. DPG is a dimensionless quantity that measures the effort exerted on the particles by the airflow, relative to their own weight. In the absence of other efforts (i.e. in the case of gravity feeding), A DPG value of 1 would correspond to the initiation of the fluidization of the bed.

In order to characterize the stability of the system, we can focus on the DPG value at the entry of the rolls: inside the press, particles are likely to be least affected due to the effort exerted by the rolls and the incoming feed. In the case of gravity feeding, DPG $>1$ will result in fluidization of the feed and process instability. In the case of screw feeding, DPG $>1$ values can be reached due to the effort exerted by the feeding system, but Johanson and Cox (1989), based on empirical considerations, stated that DPG $>10$ values are difficult to sustain even with screw feeding. In such a situation, a solution to partially eliminate air in the feed is required.

\subsection{Numerical determination of solid and fluid solutions}

Knowing the material properties of the powder and feed pressure $q_{0}$, the first step consists in determining the response of the solid under roll pressure by solving the Johanson equations. In this step, the pressure distribution $\sigma_{\theta}$ in the solid phase is first determined. The details of this step are not recalled here and can be found in various publications such as Johanson (1965) and Patel et al. (2010). Once the pressure distribution and the material compressibility $\beta$ are known, the density distribution of the solid is computed from Eq. (4), and determination of the porosity follows from Eq. (5). The last variable needed for the determination of the gas pressure in the porous medium is the velocity of the solid $v_{s}$, which is simply calculated from Eq. (8).

Once all of the aforementioned data has been collected, the gas pressure should be determined, during the roll compaction process, by solving Eqs. (12)-(15). As this problem is highly nonlinear, no analytical solution could be established, but it can be numerically integrated.

A finite differences scheme was applied for the discretization of Eq. (12), assuming an initial value for the unknown constant $B$. Compliance to the boundary condition (15) then gives us a value of $P(0)$ at the gap, and from there a numerical solution, assuming this value of $B$. In all likelihood, however, this solution does not respect boundary condition (14). The value of $B$ is then optimized through an iterative procedure until the boundary condition (14) is also met.

This numerical procedure is stable and converges rapidly. Finally, the gas pressure and its gradient are computed.

\section{Results and discussions}

\subsection{Materials and press data}

Two types of powders were used: bentonite and limestone powders. Their properties were characterized by commonly used tests in the area, and the data are summarized in Table 1. Particle size was calculated from the measure of the Blaine Specific Surface (BSS) through the relation $D_{p}=6 /(\rho \mathrm{BSS})$. This value is of course likely to differ from a characteristic size measured by another means, such as the $d_{50}$ value obtained from a measurement using laser granulometry. The measurement of the Blaine Specific Surface is based on a permeability measurement, the surface estimation coming from the Kozeny-Carman law (13a). Thus the experimental approach can alternatively be seen as measuring the permeability for a given porosity and particle size, and adopting (13a) only for the relative variations of porosity and particle size.

Simulations were carried out using a roll press diameter of $100 \mathrm{~mm}$ and a gap of $2 \mathrm{~mm}$ for bentonite, $500 \mathrm{~mm}$ and $10 \mathrm{~mm}$ for limestone.

The bentonite was considered to have a fixed particle size, and all results in the absence of particle fragmentation were recorded using this powder.

The limestone powder was characterized for its fragmentation properties. Particle size was measured for several pressures in an oedometric compression cell, a well-known solution in the grinding community for a simple characterization of powder fragmentation in compression (Fuerstenau et al., 1996; Hosten and Cimilli, 2009; Schönert, 1996). The averaged evolution of $D_{p}$, the characteristic particle size (calculated from Eq. (13)), is provided as a function of material density. The fit for this relation is represented in Fig. 2. In a characteristic pattern, the particle size quickly decreases in the early stage of compaction, before reaching a limit, as fragmentation is no longer possible in the compacted material.

\subsection{Solid pressure and porosity}

In Figs. 3 and 4 the predicted solid pressure and porosity distribution for the bentonite powder were plotted, using the Johanson approach. It was assumed that the compaction was achieved with a given maximum roll pressure of $110 \mathrm{MPa}$ and the required feed pressure qo was determined accordingly. This pressure was then used to calculate the porosity of the compact and the air pressure gradient as a function of roll rotation speeds. At the feed angle $\theta_{0}$, the porosity of the powder was relatively high (0.65) and decreased under roll pressure up to 0.2. 


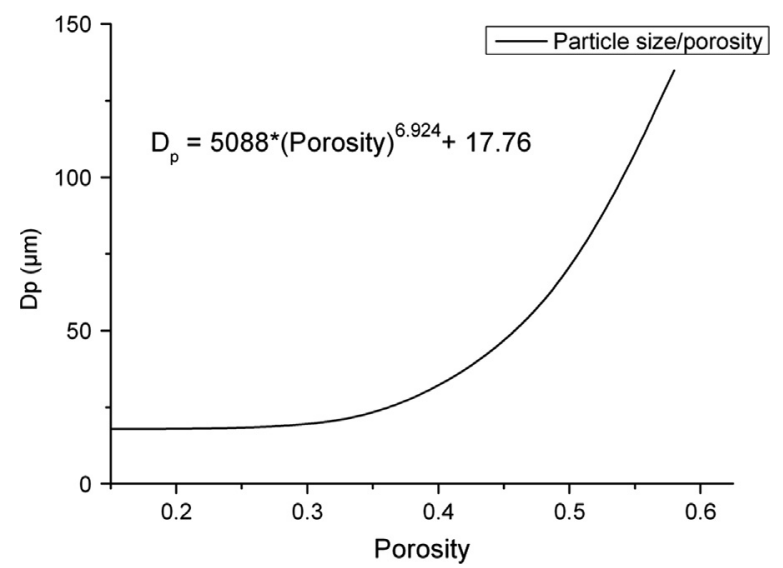

Fig. 2. Evolution of limestone particles characteristic size $\left(D_{p}\right)$ with porosity.

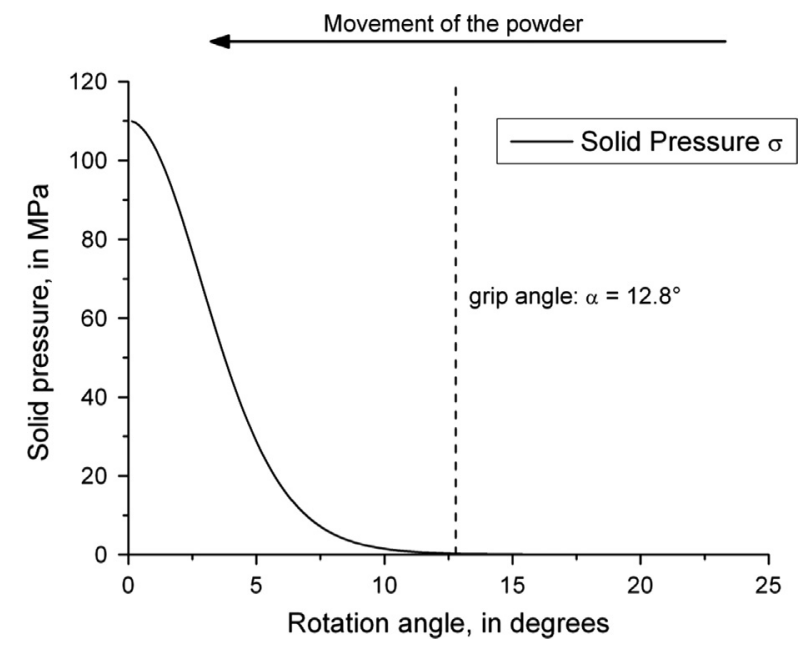

Fig. 3. Roll pressure distribution of bentonite powder (Johanson Model).

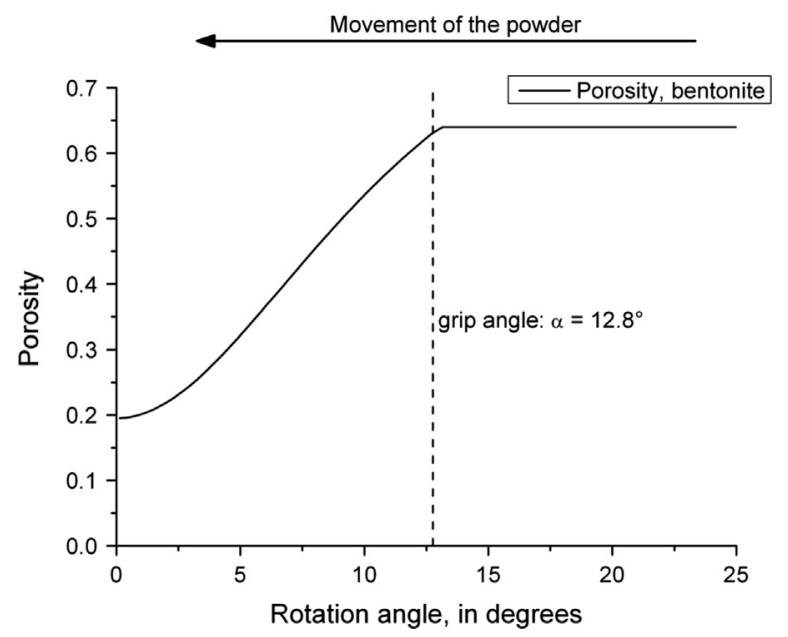

Fig. 4. Porosity distribution of the compacted bentonite powder (after Eq. (5)).

\subsection{Air pressure distribution versus roll rotation speed}

Calculated air pressure profiles are displayed in Fig. 5 for the different roll rotation speeds. Fig. 6 is a zoom on the values calculated near the entry. The higher values of the rotation speed are of course unrealistic but the results are presented to show the diversity of situations that can arise in these systems.

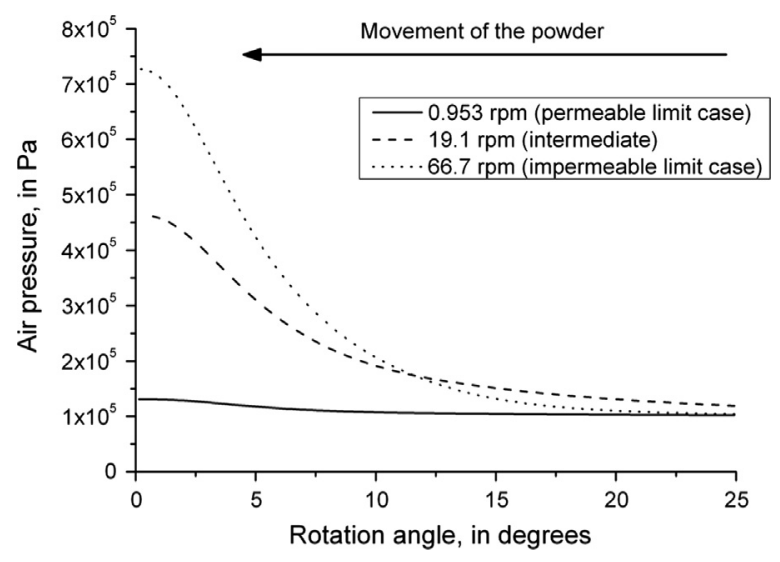

Fig. 5. Air pressure distribution versus roll rotation angle.

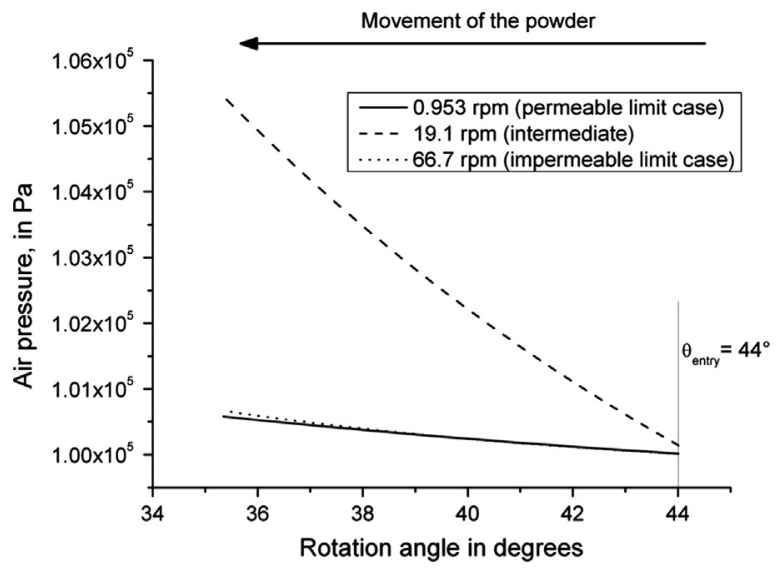

Fig. 6. Zoom on the air pressure distribution at the roll feed.

In all cases, gas pressure builds up progressively from the entry of the rolls. With the narrowing of the material slab and the densification of the powder, space available for air is reduced. Some of the air stays in the reduced space, contributing to the pressure rise, and some is evacuated through the incoming material. The air pressure profile is then going to depend on the proportion of air that is trapped in the porosity, and on the proportion that is evacuated.

Rotation speed $(0.95 \mathrm{rpm})$ is a case where the rotation speed is low, or the powder is very permeable to gas. Air migration through the powder is easy, either because the high powder permeability implies minimal resistance to gas movement, or because the slow rotation implies that the quantities of gas entrained are small. In this situation, most of the air escapes. We name this situation the "permeable limit case". Pressure build-up at the gap is minimal (Fig. 5). Pressure gradient at entry (the slope on curves in Fig. 6) is also very small, as the powder does not present significant resistance to the reduced quantity of air in movement.

Rotation speed $(66.7 \mathrm{rpm})$ embodies the opposite situation, where rotation speed is high, or the powder is very impermeable. Resistance to air movement is considerable, or the quantities of air to be evacuated are large, due to the rotation speed: most of the air is trapped in the material. We name this situation the "impermeable limit case". Pressure at the gap reaches very high values. This high pressure in the end products can eventually lead to their rupture once they are released by the press (Dec, 1995; Johanson and Cox, 1989). In a less intuitive manner, the pressure gradient at the entry is also very small. As most of gas is trapped within the porosity, the quantities of gas evacuated are not sufficient to create a high pressure gradient, at least near the entry. 
a

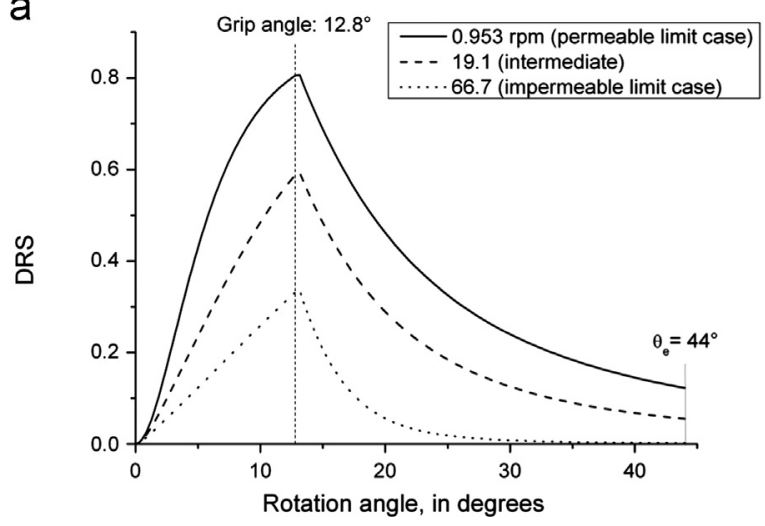

b

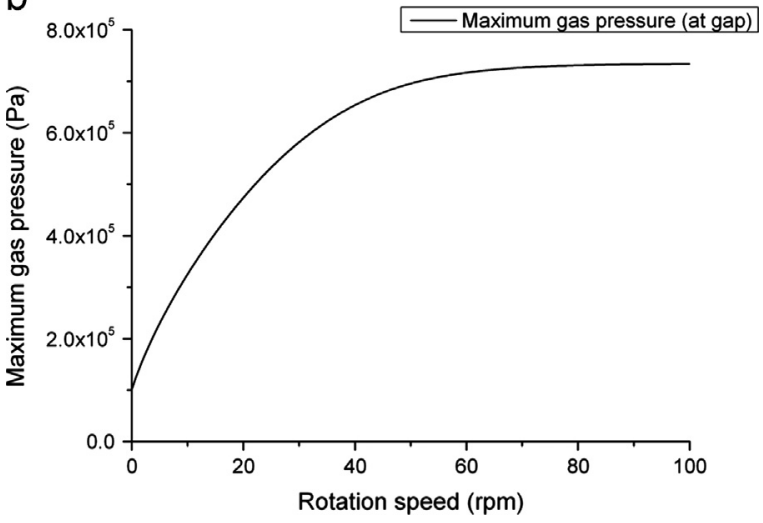

Fig. 7. (a) Dimensionless gas speed (DRS) distribution for different rolls rotation speed. (b) Maximum gas pressure (reached at the gap), as a function of rolls rotation speed (case of the bentonite powder).

As regards the gas speed, we introduce the Dimensionless Relative Speed (DRS), defined by

$\mathrm{DRS}=\left(v_{g}-v_{S}\right) / \omega R$

DRS is a dimensionless measurement of the speed of the gas relative to the moving solid as $\omega R$ corresponds to the solid speed at $\theta=0$, where it reaches its maximum.

Fig. 7a displays DRS variation with $\theta$ in the same three cases as Figs. 5 and 6. The first effect is that relative gas speed decreases with a decreasing $K / \omega$ ratio. It even tends to 0 when $K / \omega$ tends to 0 . This corresponds to increasingly impermeable conditions, as gas is forced into movement at the same speed as the solid. When $K / \omega$ tends to infinity, DRS will tend to its maximum $v_{s} / \omega R$, as the gas is not affected at all by the solid movement and stays motionless in the absolute reference frame.

We also see that the gas speed is influenced by two conflicting factors: increased confinement of the powder generates gas flow, but the compacted powder becomes impermeable to gas movement. This explains why gas speed is low at roll entry (where there is little compaction) and near the gap (where the powder is almost impermeable). The maximum reached by the DRS corresponds to the triggering of the compaction of the powder, when switching from relation (4a) to (4b) to represent powder compaction behavior. It also almost corresponds to the position of the nip angle.

Fig. $7 \mathrm{~b}$ displays the maximum gas pressure (for $\theta=0$ ) as a function of the rotation speed of the rolls. It confirms the result just stated: gas pressure rises at the gap as an increasing proportion of the air is trapped. The value reaches a limit, corresponding to the case where all the air initially present in the porosity is trapped in the compact.

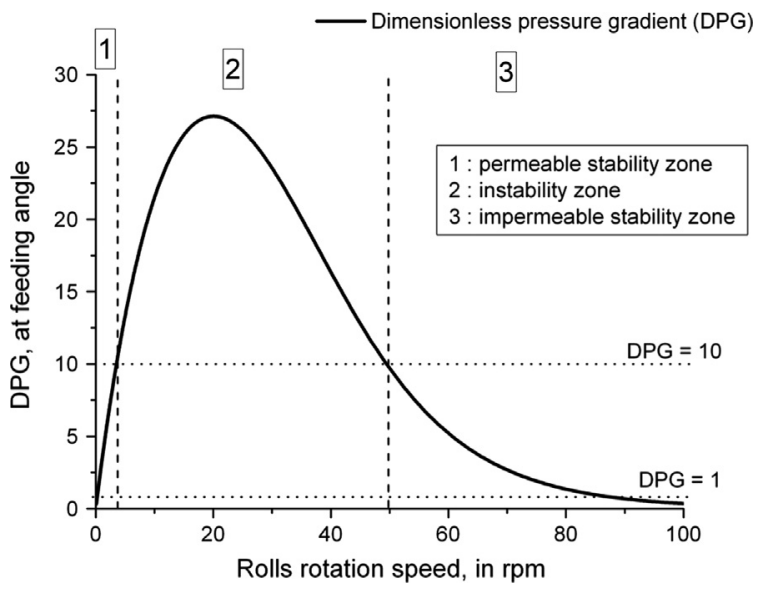

Fig. 8. Dimensionless Pressure Gradient (DPG) versus roll speed: stability zones (case of the bentonite powder).

In most applications, high gas pressure in the compacts is not acceptable, as it can reduce cohesion and damage the compacts when they are liberated from the stresses exerted by the press. Unfortunately, it is not possible to determine a general threshold value, as this would depend on the compact cohesion (and then the powder properties), as well as on the process requirements.

\subsection{Dimensionless pressure gradient and stability considerations}

In Fig. 8, the dimensionless pressure gradient DPG is plotted, calculated at the entry as a function of the rotation speed. For both extreme situations - very slow and very high rotation speed - the pressure gradient at the entry tends to 0 . The highest pressure gradients at the entry, which are the most likely to hinder the feeding system, are reached for intermediate situations, where the resistance to air is both high enough to lead to a high pressure gradient, but small enough to allow a sufficient quantity of air to be evacuated. This is the situation for Rotation speed (19.1 rpm) on Figs. 5 and 6, where the pressure gradient at the entry is close to its maximum. One can see that the maximum pressure is relatively high, but reached more progressively than in the impermeable case.

From a general point of view, dimensionless pressure gradients predicted in this simulation are very high due to the very high air content at entry. Small press dimensions are another explanation (the effect of the press dimensions is analyzed in Section 3.6).

A third point is that the proposed model can be described as conservative: as we neglect air leaking through the gap, and roll sides, we tend to overestimate air pressure building effects.

Fig. 9 is a diagram displaying isometric lines for the DPG in the rotation speed/square of particle size space. We recall that according to (13), the permeability is proportional to the square of the particle size. Unsurprisingly, when considering the role of the $K / \omega$ ratio, the isometrics are straight lines. These lines correspond to the stability thresholds defined in Section 2.2.4. They outline 2 stability zones, one corresponding to the "permeable limit case", and the other to the "impermeable case".

\subsection{Effect of particle fragmentation}

The main advantage of using expression (13) for the definition of the permeability is that it allows us to evaluate the impact of the particle fineness on the compaction process. When this fineness does not evolve with the process, we see that this impact is best assessed through consideration of the permeability/rotation speed ratio. 


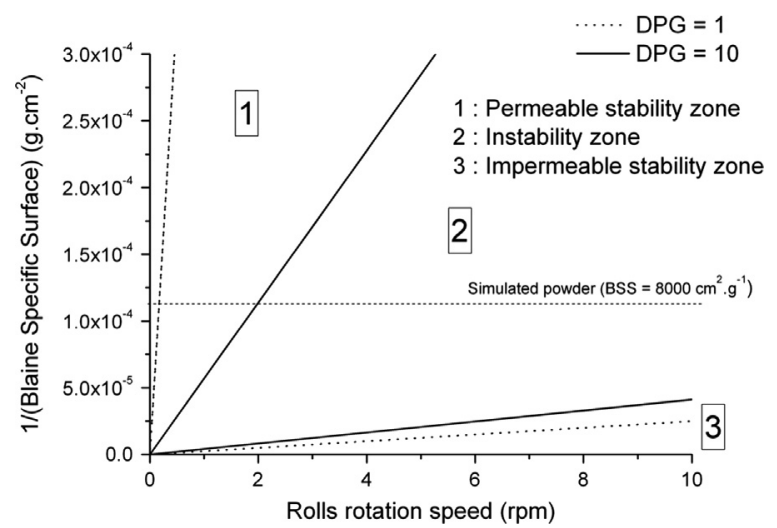

Fig. 9. Contours of Dimensionless Pressure Gradient at the entry of the rolls.

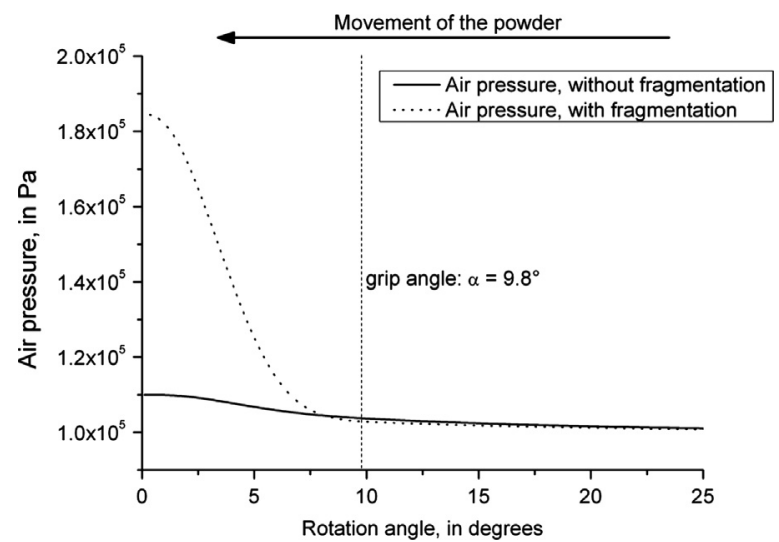

Fig. 10. Air pressure distribution. Effects of the powder fragmentation (case of the limestone powder).

We now want to consider the effect of the fragmentation of powders on the air pressure during the compaction process. This fragmentation can either be incidental, when compacting a powder where grains can display a fragile behavior (brittle material), or be the targeted outcome of the process when the roll press is used as a grinding solution. As stated in Section 3.1, we used a limestone powder whose characteristics are found in Table 1. Fragmentation is taken into account through a fitted relationship between $D_{p}$ and the porosity, which is plotted in Fig. 3. We then compare simulations where this variation of $D_{p}$ is activated to simulations where its value is kept constant at the initial value.

Including the fragmentation in the simulation exacerbates the reduction in the permeability of the powder with compaction, as the effect of the smaller particle size is added to the effect of the reduction in the porosity. This can readily be seen in Fig. 10, where we display the evolution of the permeability with the rotation angle, with and without taking into account the fragmentation. The permeabilities are initially identical, and in both cases they decrease drastically with compaction due to the reduction in porosity. But the effect is much stronger in the fragmentable case where the powder is an order of magnitude less permeable at the neutral angle.

Fig. 11 displays the consequence on the distribution of pressure. For the chosen set of conditions, we are close to the permeable limit case for reasonable values of rotation speed. The fragmentable powder clearly shows an increased tendency to entrap air in the final stage of compaction: the air pressure is much higher in the immediate neighborhood of the gap than in the absence of fragmentation. This is logical as it is in this zone that the powder permeability has been most affected by the fragmentation.

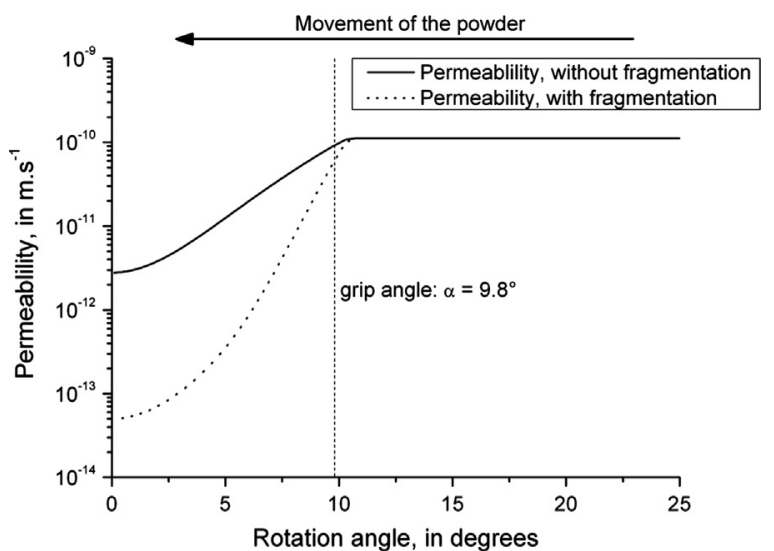

Fig. 11. Effect of the powder fragmentation on the permeability distribution (case of the limestone powder).

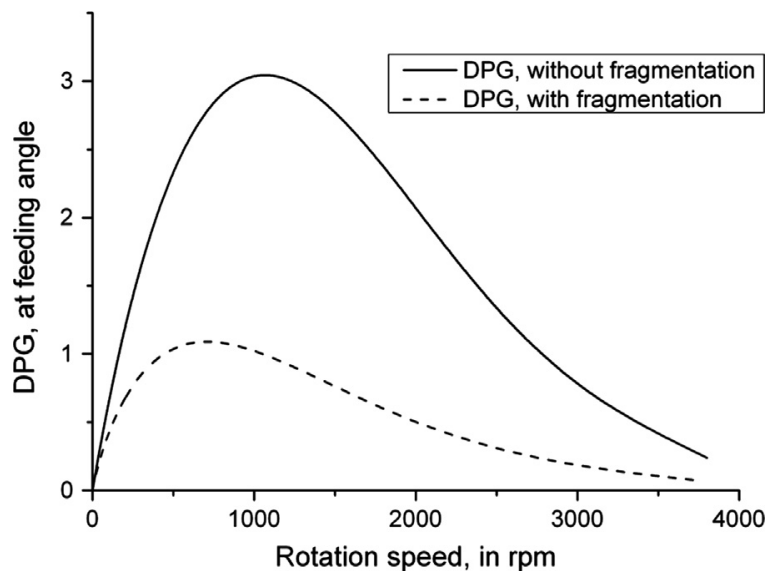

Fig. 12. Dimensionless Pressure Gradient versus roll speed. Effect of the powder fragmentation (case of the limestone powder).

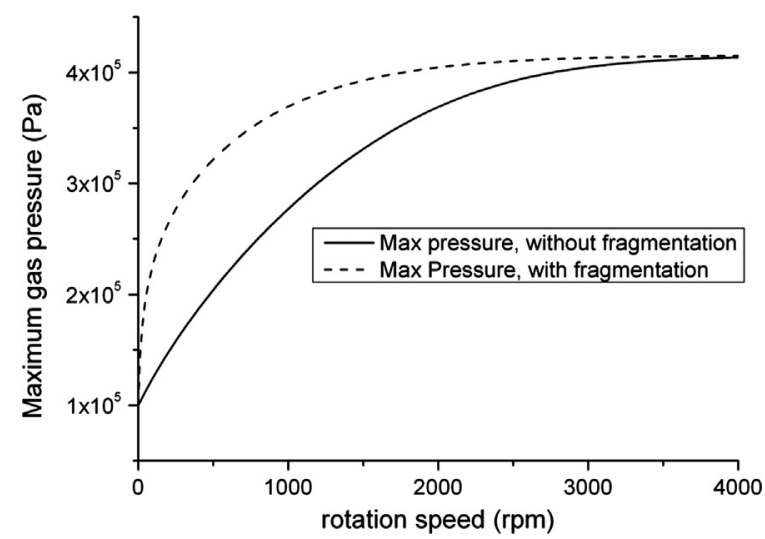

Fig. 13. Maximum gas pressure (measured at the gap) as a function of rotation speed. Limestone powder, with and without fragmentation.

The increased tendency of fragmentable powder to entrap air is also illustrated in Figs. 12 and 13, which display the DPG at the feeding angle, and the maximum pressure at the gap as a function of rotation speed. DPG is, in general, lower in the fragmentable case, and the maximum occurs for smaller values of the rotation speed. Both these features can be explained through the increased ability of the fragmentable powder to entrap air in the final stages of compaction. The maximum pressure increases faster with rotation speed for the fragmentable powder, even though in both situations the ultimate limit is the same. 
From a general point of view, it seems that the DPG at the feeding angle is only slightly affected by the fragmentation (which is very high in the chosen example). Switching from a nonfragmentable to a fragmentable powder with similar initial fineness could have only a limited impact on process stability. If the preoccupation is more about the maximum air pressure in the solid (to avoid explosion of the solid when liberated by the rolls, for instance), the effect is much stronger, even at low rotation speeds.

\subsection{Dimensional scale-up and effects on gas pressure}

The size of compaction installations can vary dramatically with roll diameters varying, for instance, from about $10 \mathrm{~cm}$ for small lab-scale installations to $2 \mathrm{~m}$ for the biggest presses used for grinding in the mineral industry. In the Johanson model, as in most simulations where only the stress in the solid phase is calculated, the size of the press does not have a direct impact on the calculated roll pressure. The latter only depends on the geometry through dimensionless ratios, such as the gap width/ roll diameter ratio, the entry angle, etc. But this is no longer the case when considering the calculation of air pressure and its gradient.

We have chosen to focus on the air gradient at the entry, which we know is of special interest for the stability of the system.

We examined, from the studied model (Eq. (16)), the situation where all spatial dimensions are amplified by a factor $\lambda$ : $(\bar{x}=\lambda x, \bar{R}=\lambda R, \bar{S}=\lambda S)$. With these assumptions we now want to see for which rotation speed $\bar{\omega}$ we could have the same air pressure distribution: $P(\theta, \omega)=\bar{P}(\theta, \bar{\omega})$.

We designate $\bar{P}(\bar{x})$ the resulting air pressure from Eq. (16) for a roll rotation speed $\bar{\omega}$. After considering that $\frac{\partial \bar{P}}{\partial \bar{x}}=\frac{1}{\lambda} \frac{\partial P}{\partial x}$, it can then readily be seen that for $\bar{\omega}=\frac{\omega}{2}$, pressures $P$ and $\bar{P}$ satisfy the same equation (Eq. (16)). This means that for $\left(\omega, \frac{\partial P}{\partial x}\right)$ and $\left(\bar{\omega}=\frac{\omega}{\lambda^{2}}, \frac{\partial \bar{P}}{\partial \bar{x}}=\frac{1}{\lambda} \frac{\partial P}{\partial x}\right)$, pressure profiles $P$ and $\bar{P}$ as functions of the rotating angle (which is the same at any scale) are identical. The effect of a change in the dimensions of the system can then be deduced for the pressure field and its gradient through a very simple transformation.

This simple result could be of prime importance for compaction system design at different scales, if the model could be validated by experiments. To our knowledge, this finding has never been presented in the literature.

The first consequence of this finding mainly concerns the scaleup from laboratory press to the pilot or to the production presses. By doubling the size of the press and the gap, and keeping their ratio constant, for example, the DPG at the feeding angle as a

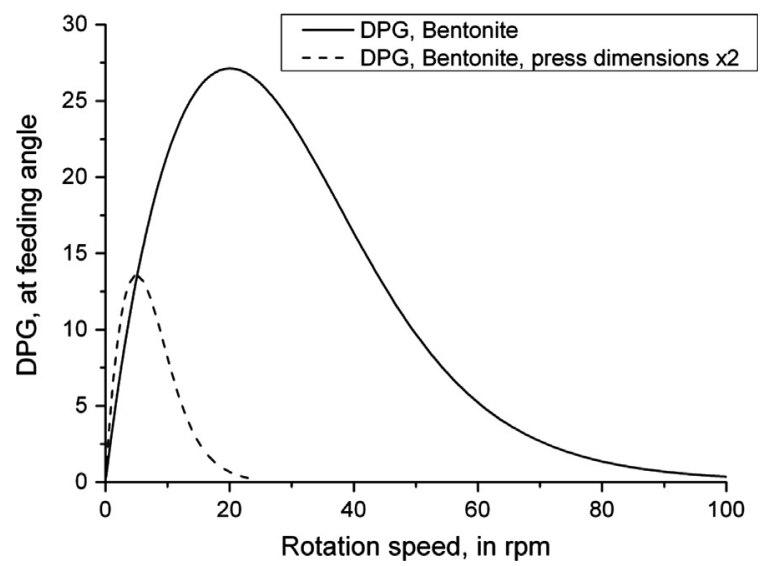

Fig. 14. Dimensionless Pressure Gradient at feeding angle. Effect of press dimension. function of the roll rotation speeds, we can take profiles like those plotted in Fig. 14.

As regards the maximum pressure at the gap, the effect is clear: presses of different dimensions will generate similar pressure distributions for rotation speeds proportional to $\frac{1}{\lambda^{2}}$. Bigger presses are always going to generate much higher-pressure build-up for similar rotation speeds.

As concerns the pressure gradient at the entry, at first sight, an increase of the system dimension seems to be a disadvantage for the stability of the system, at least near the permeable limit case, where most industrial systems seem to function. The DPG takes somewhat smaller values, but they are reached for even smaller values of the rotation angle. The DPG is only going to reach lower values for bigger systems as they function close to their maximum DPG or in the impermeable limit case. However, a bigger press can provide considerable throughput even for small rotation speeds (throughput is proportional to $\lambda^{3} \omega$ ). This could compensate for the limitation in terms of rotation speed.

These considerations would of course benefit considerably from being confirmed by experimental studies of the behavior of presses of different sizes. We believe, however, that they are valuable to anyone involved in the design of a compaction system affected by air evacuation, and its scale-up.

\section{Conclusion}

A 1D model for air flow during powder roller compaction was proposed and tested, based on the Johanson model for calculation of the solid stress and deformation, and on Darcy's law for fluid transport. Permeability was set as a function of porosity and particle size through the use of the Kozeny-Carman law. In this modeling, two assumptions were assumed: the effect of the air pressure on the solid was neglected between rolls and the airflow through the gap was ignored. We stress the importance of the ratio between permeability and roll rotation speeds, which determines the proportion of air trapped within the compact. According to the variations of this parameter, the model predicts the existence of two stability zones where industrial systems could possibly be operated, unaffected by the effect of air transport through the feed. The effects of the fragmentation of the particles were also investigated. Finally, the model provides guidelines for compaction systems scale-up, which could prove useful for dimensioning systems subjected to important air elimination issues.

The proposed model is a considerable simplification, and does not allow us to predict all of the subtleties of the fluid flow. The present study would also benefit greatly from the experimental validation.

\section{Nomenclature}

$\theta \quad$ rotating angle

$x=R \sin \theta$ axis of rolls symmetry

$D=2 R \quad$ roll diameter

$S \quad$ gap

$\omega \quad$ rotational roll speed

$\rho_{\theta}, \rho_{s} \quad$ apparent and true density of solid

BSS Blaine Specific Surface

g gravity $\left(\mathrm{m}^{2} / \mathrm{s}\right)$

$\varphi_{w}, \delta \quad$ wall and internal friction angle

$\sigma_{\theta} \quad$ mean normal stress at position $\theta$

$\sigma_{i}(\theta), i=1,2$ major and minor principal stresses

$q_{0}, \sigma_{0}, \theta_{0}$ pressure, normal stress and feed angle

$\phi, v_{s} \quad$ porosity and solid velocity 


$\begin{array}{ll}\beta & \text { compressibility coefficient of powder } \\ \rho_{g} & \text { density of the gas } \\ v_{g} & \text { superficial velocity of gas } \\ K & \text { permeability } \\ \mu_{g} & \text { gas viscosity } \\ D_{p} & \text { average size of particles } \\ P & \text { gas pressure } \\ \text { DPG }=\frac{\partial P}{\partial x} / \rho_{s} g \text { Dimensionless Pressure Gradient at the entry } \\ R_{e} \quad \text { Reynold's number } \\ \text { DRS } \quad \text { Dimensionless Relative Speed }\end{array}$

\section{References}

Coffey, K.A., Gremaud, P.A., 2003. Numerical simulation of aerated powder consolidation. Int. J. Non-Linear Mech. 38, 1185-1194.

Coussy, O., 2004. Poromechanics. John Wiley and Son's, Ltd., England.

Cunningham, J.C., 2005. Experimental Studies and Modeling of the Roller Compaction of Pharmaceutical Powders. Drexel University. (Ph.D. thesis). 〈http:/ dspace.library.drexel.edu/bitstream/1860/837/1/Cunningham_John.pdf).

Cunningham, J.C., Windstead, D., Zavaliangos, A., 2010. Understanding variation in roller compaction through finite element-based process modelling. Comput. Chem. Eng. 34, 1058-1071.

Bindhumadhavan, G., Seville, J.P.K., Adams, M.J., Greenwood, R.W., Fitzpatrick, S, 2005. Roll compaction of a pharmaceutical excipient: experimental validation of rolling theory for granular solids. Chem. Eng. Sci. 60, 3891-3897.

Dec R.T., 1995. Problems with processing of fine powders in roll press, In: Proceedings of the 24rd Biennial Conference of the Institute for Briquetting and Agglomeration, vol. 24, pp. 199-210.

Dec, R.T., Zavaliangos, A., Cunningham, J.C., 2003. Comparison of various modeling. methods for analysis of powder compaction in roller press. Powder Technol. 130, 265-271.

Dullien, F.A.L., 1992. Porous Media Fluid Transport and Pore Structure. Academic press, San Diego.

Fuerstenau, W., Gutsche, O., Kapur, P.C., 1996. Confined particle bed comminution under compressive loads. Int. J. Miner. Process. 44-45, 521-537.
Hosten C., Cimilli H., 2009, The effects of feed size distribution on confined-bed comminution of quartz and calcite in piston-die press, Int. J. Miner. Process., 91, 3-4.

Jenike, A.W., Shield, R.T., 1959. on the plastic flow of Coulomb solids beyond original failure. J. Appl. Mech. 26, 599-602.

Jenike, A.W., Elsey, P.J., Woolley, R.H., 1960. Flow properties of bulk solids. ASTM Proceedings $60,1168-1181$.

Johanson, J.R., 1965. A rolling theory for granular solids. ASME, J. Appl. Mech. 32, 4. Johanson, J.R., 1971. Modeling flow of bulk solids. Powder Technol. 5, 93-99.

Johanson, J.R., Jenike, A.W., 1971. The effect of gaseous phase on pressures in a cylindrical silo. Powder Technol. 5, 133-145.

Johanson, J.R., Cox, B.D., 1989. Fluid entrainment effects in roll press compaction. Powder Handl. Process. 1, 2.

Hsu, S.-H., Reklaitis, G.V., Venkatasubramanian, V., 2010. Modeling and control of roller compaction for pharmaceutical manufacturing: part I: process dynamics and control framework. J. Pharm. Innovation 5, 14-23.

Michrafy, A., Diarra, H., Dodds, J.A., Michrafy, M., 2011a. Experimental and numerical analyses of homogeneity over strip width in roll compaction. Powder Technol. 206, 154-160.

Michrafy, A., Diarra, H., Dodds, J.A., Michrafy, M., Penazzi, L., 2011b. Analysis of stress strain rate in roller compaction process. Powder Technol. 208, 417-422.

Muliadi, A.R., Lister, J.D., Wassgren, C.R., 2012. Modelling the powder roll compaction process: comparison of 2-D finite element method and the rolling theory for granular solids. Powder Technol. 221, 90-100.

Murfitt, P.G., Bransby, P.L., 1980. Deaeration of powders in hoppers. Powder Technol. 27, 149-162.

Patel, B.A., Adams, M.J., Turnbull, N., Bentham, A.C., Wu, C.-Y., 2010. Predicting the pressure distribution during roll compaction from uniaxial compaction measurements. Chem. Eng. J. 164, 410-417.

Peter, S., Lammens, R.F., Steffens, K.-J., 2010. Roller compaction/Dry granulation: Use of the thin layer model for predicting densities and forces during roller compaction. Powder Technol. 199, 165-175.

Sommer, K., Hauser, G., 2003. Flow and compression properties of feed solids for roll type presses and extrusion presses. Powder Technol. 130, 272-276.

Schönert, K., 1996. influence of particle bed configurations and confinements on particle breakage. Int. J. Miner. Process. 44-45, 1-16.

Schwechten, D., Milburn, G.H., 1990. Experiences in dry grinding with high compression roller mills for end product quality below $20 \mu \mathrm{m}$. Miner. Eng. 3 (1-2), 23-34

Vinogradov, G.A., Fedorchenko, I.M., 1961. Poroshk. Metall. 1 (1), 61. 